\title{
Characterization of Ultra-Thin Composite Triangular Rollable and Collapsible Booms
}

\author{
Christophe Leclerc, ${ }^{*}$ Lee Wilson, ${ }^{*}$ Miguel A. Bessa ${ }^{\dagger}$ and Sergio Pellegrino ${ }^{\ddagger}$ \\ California Institute of Technology, 1200 E California Blvd Pasadena, CA 91125
}

\begin{abstract}
The paper studies the behavior of Triangular Rollable and Collapsible (TRAC) booms made from ultra-thin carbon fiber, with a total flange thickness of $71 \mu \mathrm{m}$. Both bending and torsional behavior of the deployed booms are studied using numerical analysis and experimental testing. The coiling of the booms around hubs of large radius is also studied.
\end{abstract}

\section{Nomenclature}

$d_{\text {end }}$ Displacement of TRAC boom end

$D_{i i} \quad$ Elements of D matrix

$R \quad$ Coiled radius of curvature

$r \quad$ Flange radius of curvature

$t \quad$ Flange thickness

$w \quad$ Bonded region width

$\alpha \quad$ Angle of localized bend

$\kappa_{l} \quad$ Longitudinal curvature

$\kappa_{t} \quad$ Transverse curvature

$\theta \quad$ Flange opening angle

\section{Introduction}

Deployable booms are used to support large aperture spacecraft structures, such as solar sails, photovoltaic panels and antennas. The Triangular Rollable and Collapsible (TRAC) boom, ${ }^{1}$ which consists of two tape springs joined together along one edge, is a particular type of deployable boom with many attractive features, including high ratio of deployed bending stiffness to packaged height when compared to other types of structures that can be flattened and coiled, such as the the Storable Tubular Extendable Member or the lenticular mast. ${ }^{2}$ TRAC booms have been used in several cubesat designs, ${ }^{3,4}$ where the main attractions were their low mass, high packaging efficiency and ability to self-deploy.

Figure 1 shows the TRAC boom architecture and its packaging behavior. The main design parameters are the flange radius of curvature $r$, thickness $t$, opening angle $\theta$ and the bonded section width $w$.

Previous characterizations of the mechanical properties of deployed TRAC booms have focused on booms made from carbon fiber composite with flange thickness of $\sim 1 \mathrm{~mm} .^{2}$ The aim of this study is to extend the range of TRAC boom applications by considering boom with much thinner flanges, in order to reduce the packaged volume and thus enable longer booms on small spacecraft. Results will be presented for the specific case of TRAC booms with a flange thickness of only $71 \mu \mathrm{m}$, made from ultra-thin carbon fiber composite. These thinner TRAC booms are also interesting as potential enablers of deployable space structures of different scales.

\footnotetext{
${ }^{*}$ Graduate Student, Graduate Aerospace Laboratories, California Institute of Technology, 1200 E California Blvd. MC $205-45$

${ }^{\dagger}$ Postdoctoral Scholar, Graduate Aerospace Laboratories, California Institute of Technology, 1200 E California Blvd. MC $105-50$

${ }^{\ddagger}$ Joyce and Kent Kresa Professor of Aeronautics and Professor of Civil Engineering, Graduate Aerospace Laboratories, California Institute of Technology, 1200 E California Blvd. MC 105-50. AIAA Fellow. e-mail: sergiop@caltech.edu
} 


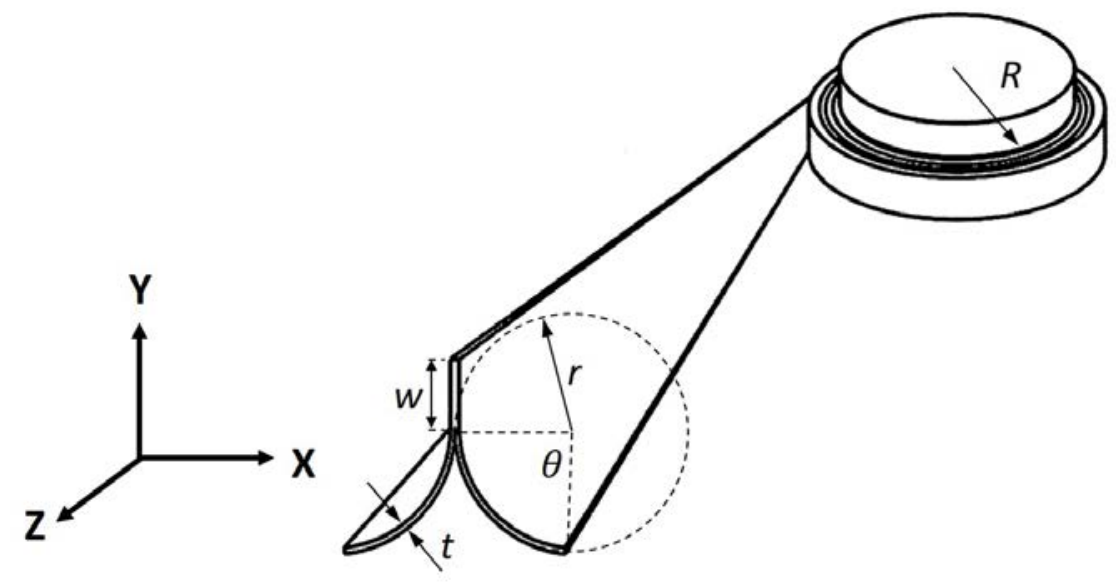

Figure 1. TRAC architecture (modified from Banik and Murphey ${ }^{2}$ )

Another topic that has been investigated in the present study is the coiling behavior of TRAC booms. Both standard tape springs and TRAC booms have the property that they form localized bends of uniform radius. For isotropic tape springs, this radius is equal to the transverse radius of curvature, $r$, of the tape spring. Hence, tape springs can easily be tightly wrapped around any radius $\simeq r$. However, when they are coiled around radii $R \gg r$, tape springs form multiple localized bends ${ }^{5}$ instead of conforming to the coil radius. Murphey and Banik ${ }^{1}$ noted that a similar effect occurs in TRAC booms, as the inner flange in a wrapped boom has a tendency to bulge inwards, which causes non-smooth coiling. The effect of applying a small tension force to stabilize this behavior will be explored, and it will be shown that in ultra-thin booms the required force is quite small.

This effect is studied using finite element analysis (FEA), by extending established approaches to modeling tape spring behavior. Previous FEA studies have included determining the bending stiffness of deployed booms, the buckling load ${ }^{6,7}$ and even the coiling of a carbon fiber tape spring around a hub, ${ }^{8}$ where $R \simeq r$. In this study, a combination of LS-Dyna FEA simulations with an experimental validation is used to capture the tension force required to coil a TRAC boom around a hub with $R \gg r$, as well as the intermediate configurations the boom forms.

\section{Manufacturing Process}

Ultra-thin composite TRAC booms were manufactured from a 17 GSM uni-directional tape supplied by North Thin Ply Technology (fibers T800 and epoxy resin ThinPreg 120EPHTg-402). This material has a cured thickness of about $18 \mu \mathrm{m}$ per ply. As the nominal diameter of the T800 filaments is $5 \mu \mathrm{m}$, the prepreg material only has a few fibers across the ply thickness. As a result, small defects and fiber misalignment have a sizable effect on the post-cure shape of the part.

A three part mold was used, as shown in Figure 2(a). The two $U$ shape components are made of aluminum, while the top part is made of silicone, allowing pressure to be applied in the $V$ groove. This allows the TRAC boom to be manufactured in a single step, instead of manufacturing two tape springs and then bonding them together in a subsequent step. It was observed that this method leads to a stronger bond between the two flanges, reducing delamination problems at the interface. Figure 2 illustrates the different steps of this manufacturing process. First, the layup is wrapped on each of the aluminum molds. Then, the molds are clamped together and the silicone part inserted into the $V$ groove. In this configuration the part is vacuum bagged and cured in an autoclave. The final TRAC boom is then cut from the resulting composite part, as illustrated in Figure 2(c) and 2(d). Different layups were studied, but the most promising stacking sequence is $[0 / 90]_{S}$, in order to keep the thickness as small as possible.

The following nominal geometric parameters were chosen: $r=12.5 \mathrm{~mm}, \theta=90^{\circ}$ and $w=8 \mathrm{~mm}$. As the prepreg material is ultra-thin, residual stresses in the cured parts induce a deformation of the final part. A FaroArm 3D scanner was used to measure the exact shape of the final TRAC booms, leading to the following geometric parameters: $r=10.6 \mathrm{~mm}, \theta=105^{\circ}$ and $w=8 \mathrm{~mm}$. A precise estimation of the thickness was 


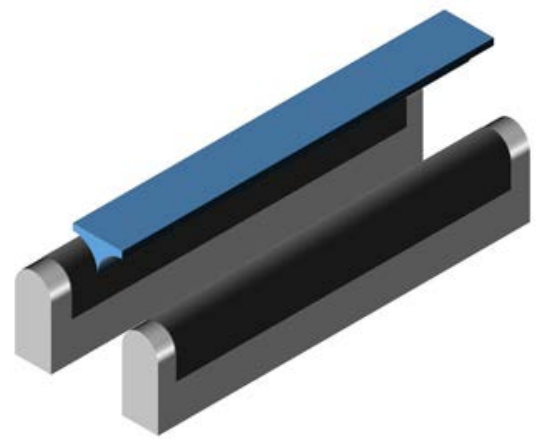

(a) Exploded view

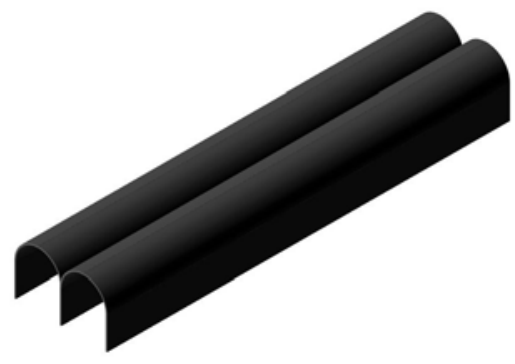

(c) Cured part

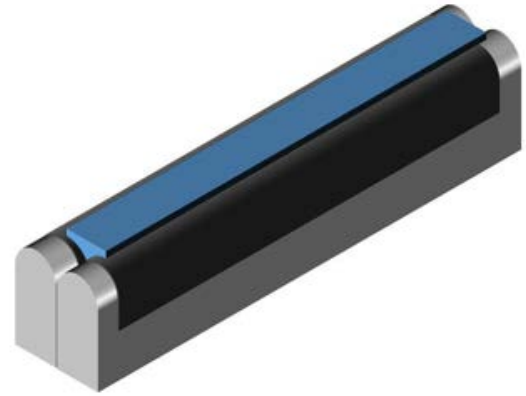

(b) Curing configuration

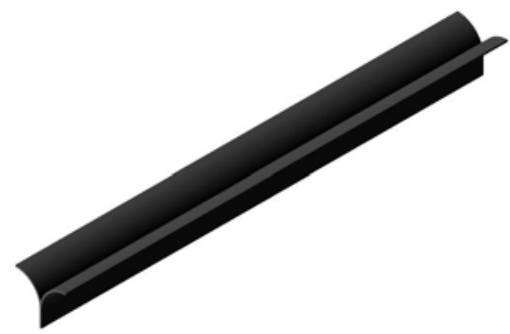

(d) Final structure

Figure 2. TRAC boom manufacturing process.

obtained using micrographs of the TRAC boom cross section. Figure 3(a) is micrograph of a single flange (4 plies) and Figure 3(b) is a micrograph of the bonded region (8 plies). Based on multiple samples, the measured flange thickness is approximately $71 \mu \mathrm{m}$ and the bonded section thickness approximately $200 \mu \mathrm{m}$.

Using the same apparatus, the transition region between the bonded section and the two flanges was measured. Figure 4 shows two examples of such region. In the first micrograph, the fibers are distorted, creating a blunt transition. This led to a thicker region where strains are higher during coiling of the TRAC boom. In the second, the two flanges are not distorted which created a sharper transition.

\section{Deployed Behavior}

As each specific application of the TRAC boom will lead to a different loading condition, both bending and torsional behavior were characterized. The properties were predicted by finite element analysis and validated through experiments. The stiffness was obtained both for bending and torsion, and the buckling loads were obtained for bending only.

\section{III.A. Simulations}

The commercial finite element software (ABAQUS/Standard) was used to predict the deployed behavior of the TRAC booms. Figure 5 shows the finite element model and mesh, composed of reduced integration four-node quadrilateral thin-shell elements. One end (End A) was kept clamped throughout the simulation, while the nodes at the other end (End B) were tied together to create a rigid cross-section. Rotation was enforced at End B, and the reaction moment obtained. Four different load cases were considered: bending around $\mathrm{Y}$, bending around $\mathrm{X}$ toward the opening (negative moment at End B), bending around X away from the opening (positive moment at End B) and torsion. The analysis procedure to predict the buckling and postbuckling behavior of the structure under bending moment follows four steps:

1. Preliminary prediction of the buckling moment by a linear perturbation (linear eigenvalue) buckling analysis of the undeformed structure;

2. An implicit static analysis was conducted up to the predetermined buckling moment or until the analysis stopped due to buckling; 


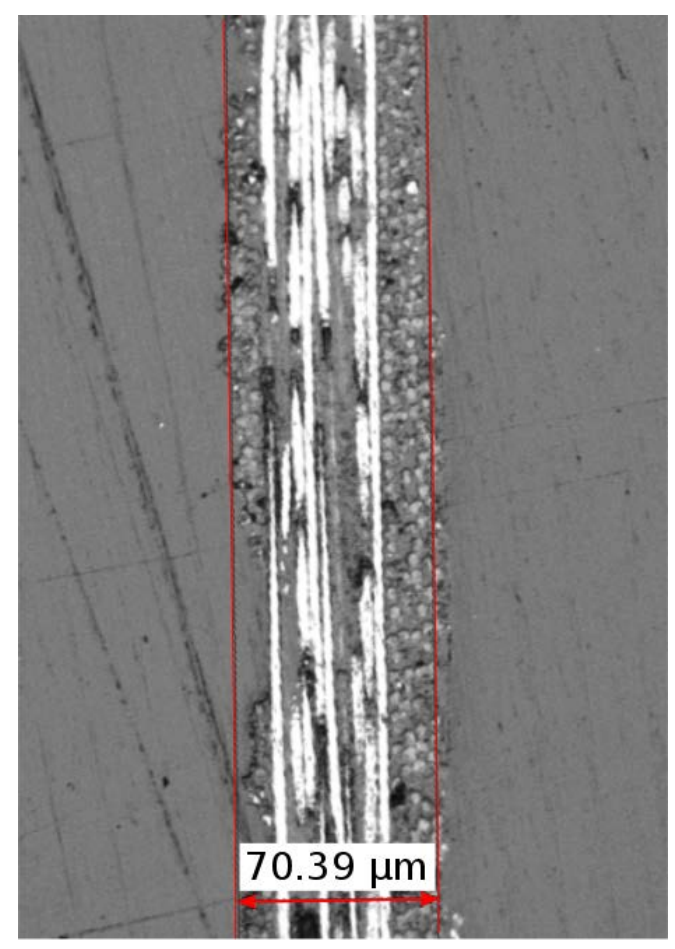

(a) Single flange

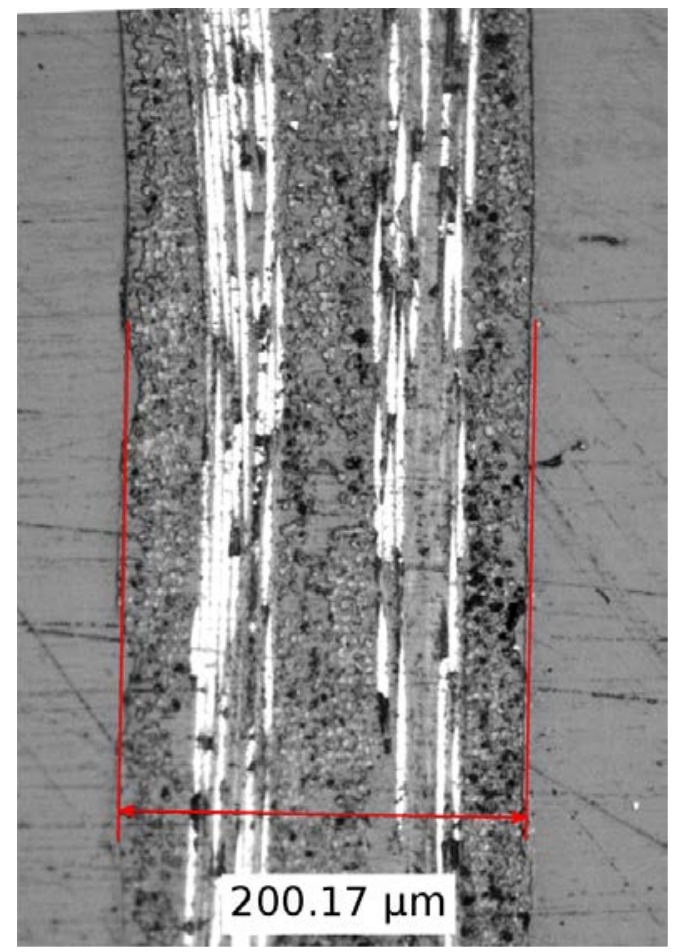

(b) Bonded region

Figure 3. Micrographs of TRAC boom cross section.

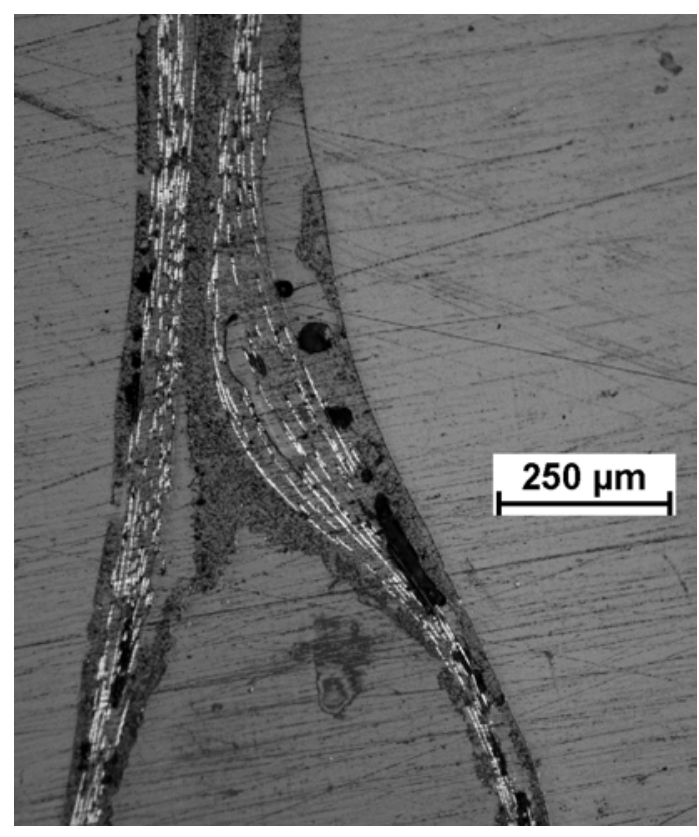

(a) Blunt transition

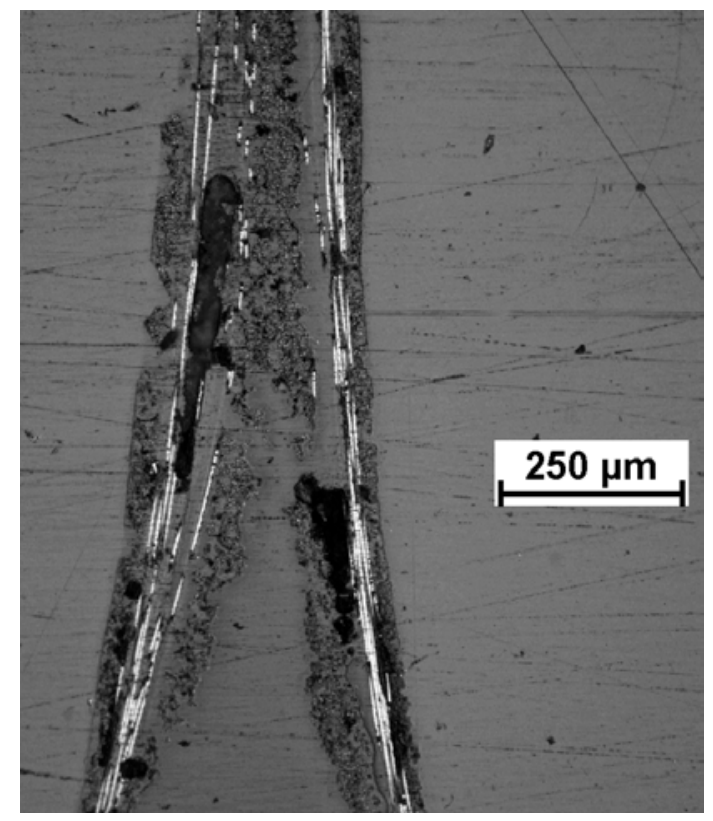

(b) Sharp transition

Figure 4. Micrographs of TRAC boom transition region. 
3. Linear perturbation buckling analyses performed sequentially from the last increment of the implicit static simulation until a previous step where the buckling moment could be determined. This sequential procedure was used because the last increments of the implicit static analysis were usually already in a postbuckling regime, where the eigenvalue buckling analyses are not valid;

4. Postbuckling analysis using the modified RIKS method and introducing an imperfection in the geometry of the structure as a combination of the buckling modes determined in step 3. The predictions shown in the subsequent figures were obtained for an imperfection that considered the first buckling mode with an amplitude of $10 \%$ of the laminate thichness $(7.1 \mu \mathrm{m})$. Other combinations of buckling modes were considered as imperfections without a significant difference in the buckling prediction of the buckling and postbuckling behavior. This is attributed to the fact that the first 10 buckling modes determined for the idealized structure occur at similar buckling moments and in a highly localized region near the center of the TRAC booms.

The torsional behavior is obtained using only an implicit nonlinear static analysis, as there is no buckling in the range that we are interested in.

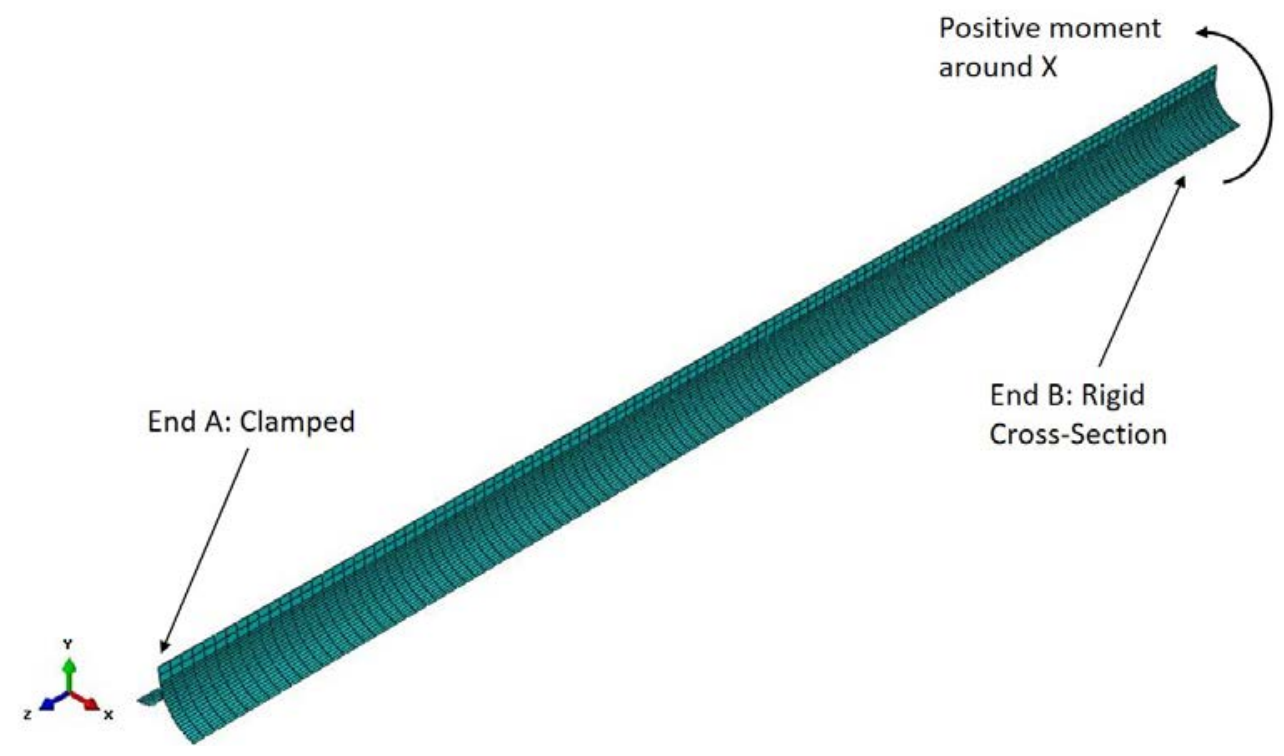

Figure 5. TRAC boom finite elements model

\section{III.B. Experimental Setup}

The mechanical properties were obtained experimentally using two different setups. The first setup, shown in Figure 6, uses gears to rotate both ends of the structure, while strain gauges mounted on hollow shafts measured the moment applied at each end. The rotation at each end was measured using photogrammetry. The boom is clamped at both ends in 3D-printed blocks and held into place using epoxy resin. These end fixtures have been designed to allow bending tests around both $\mathrm{X}$ and $\mathrm{Y}$ for the same sample. Their geometry was tailored for each sample based on the data obtained from the 3D scanner. The rotation angle was increased at both ends in order to keep the moments equal, until buckling occurred. The bending stiffness was computed from

$$
E I=\left(\frac{d M}{d \theta}\right) L
$$

where the derivative was obtained through linear regression of the experimental data. Three samples were tested, but the results of sample 1 were rejected as a crack was noticed. This sample had lower stiffness and buckling load.

The second setup, shown in Figure 7, used a gearbox at one end to control the torsion angle of the structure while a load cell (ATI Nano17 force/torque sensor) measured the torque at the other end. The TRAC boom was clamped at both ends in 3D-printed blocks that were specifically designed in order to align 


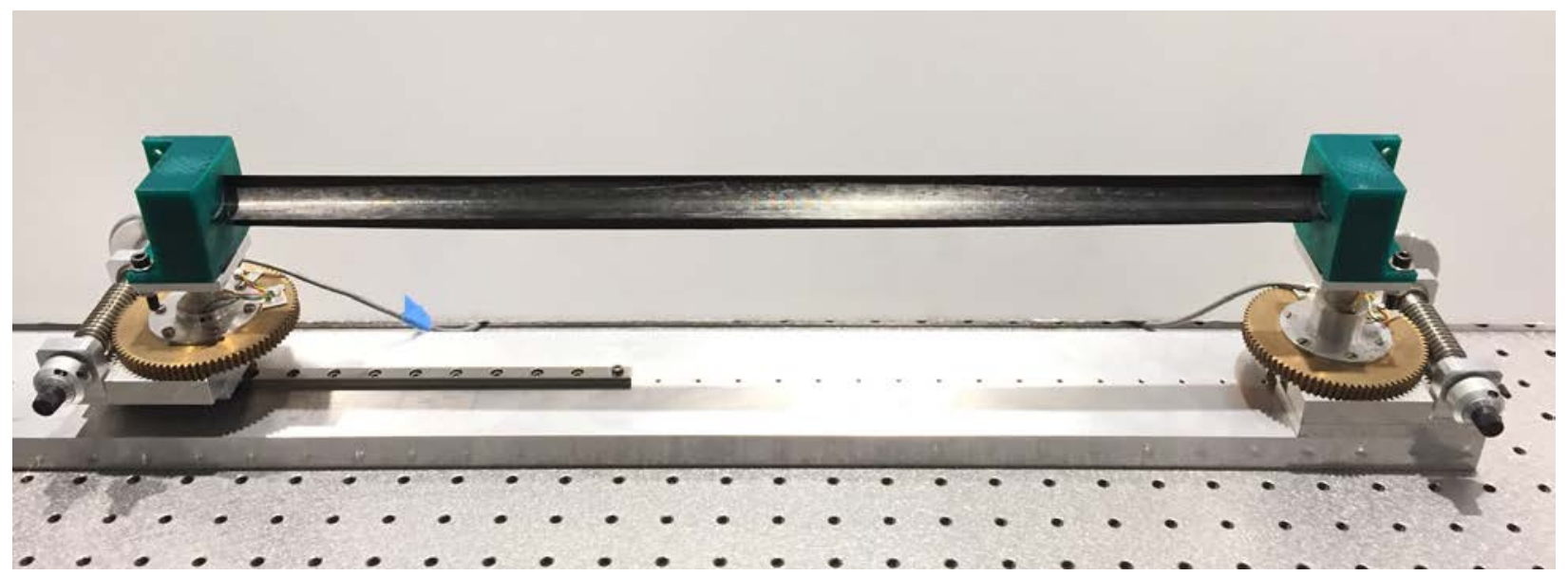

Figure 6. Bending experimental setup

the centroid of the structure with the rotation axis of the gearbox. The torsional stiffness can be extracted from the moment-angle curve using

$$
G J=\left(\frac{d M}{d \theta}\right) L
$$

As the torsional behavior is non-linear, the derivative was obtained by linear regression over a range of $5^{\circ}$. The experiment was stopped when small localized buckling appeared close to one end of the boom. Only one sample was tested in torsion.

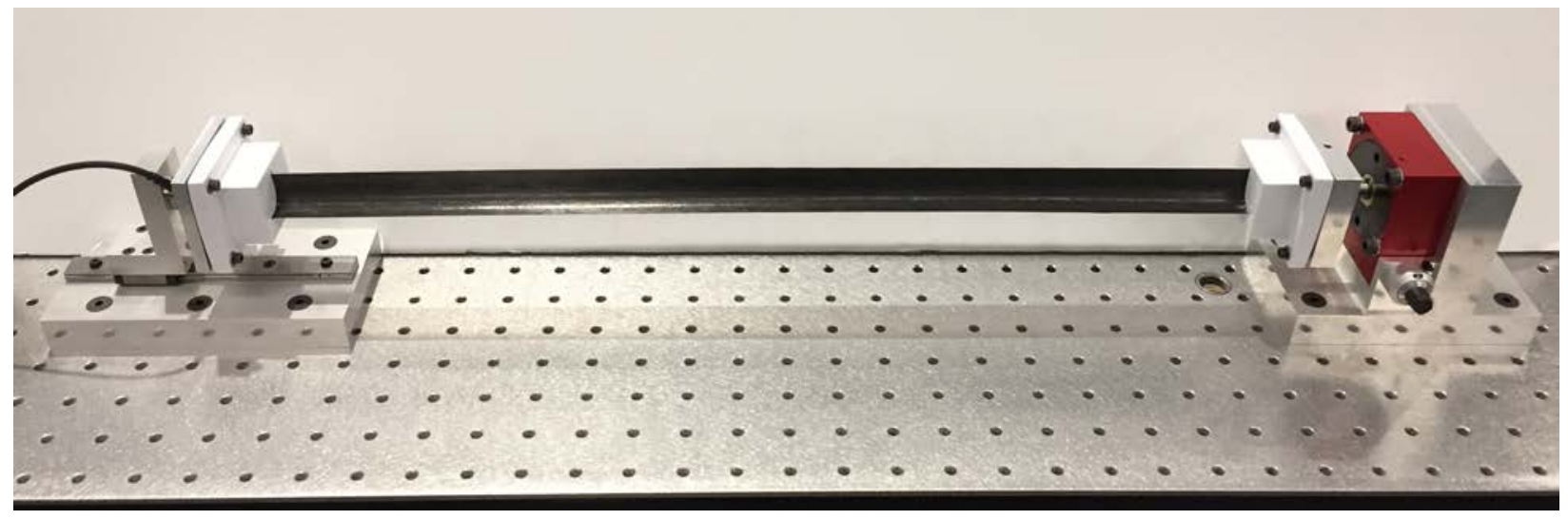

Figure 7. Torsion experimental setup

\section{III.C. Results}

The results from the tests and simulations are presented in Figures 8 and 9. Table 1 summarizes the values of the bending and torsional stiffnesses; the bending stiffness is an average of two experiments.

Table 1. Bending and torsional stiffness of deployed boom

\begin{tabular}{l|c|c} 
& Simulation & Experiment \\
\hline \hline Bending Stiffness in $\mathrm{X}\left(\mathrm{N} \mathrm{m}^{2}\right)$ & 8.91 & 8.15 \\
\hline Bending Stiffness in $\mathrm{Y}\left(\mathrm{N} \mathrm{m}^{2}\right)$ & 7.94 & 3.32 \\
\hline Bending Stiffness in $\mathrm{Y}\left(\mathrm{N} \mathrm{m}^{2}\right)$ (Post Buckling) & 3.42 & 3.32 \\
\hline Torsional Stiffness $\left(\mathrm{N} \mathrm{m}^{2}\right)$ & 0.00071 & 0.0013 \\
\hline \hline
\end{tabular}




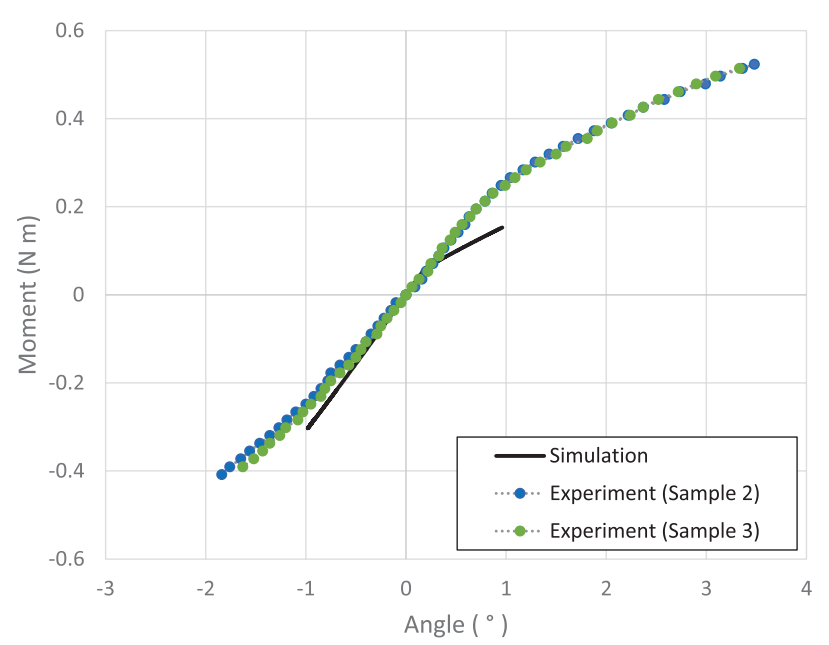

(a) Bending around $\mathrm{X}$

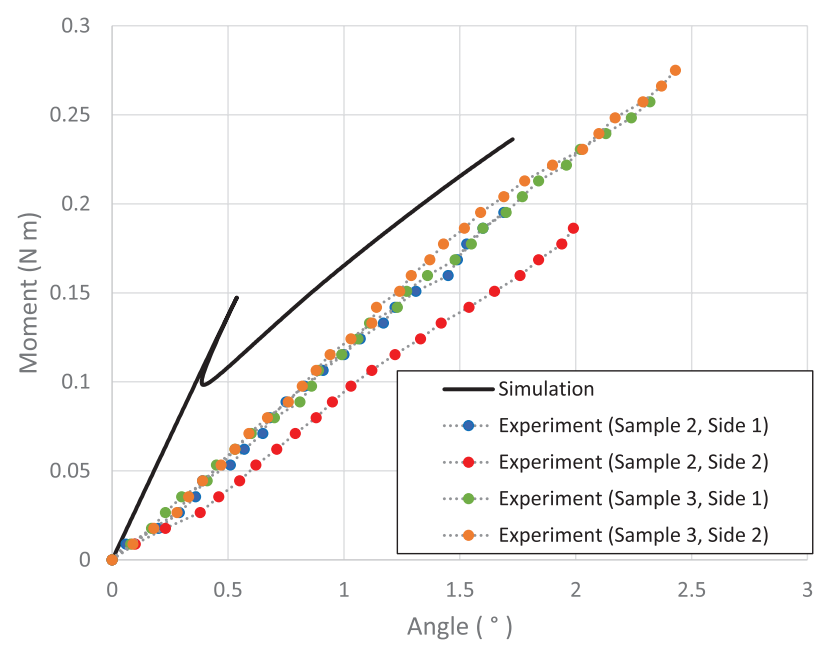

(b) Bending around $\mathrm{Y}$

Figure 8. Comparison of bending results from simulations to experiment.

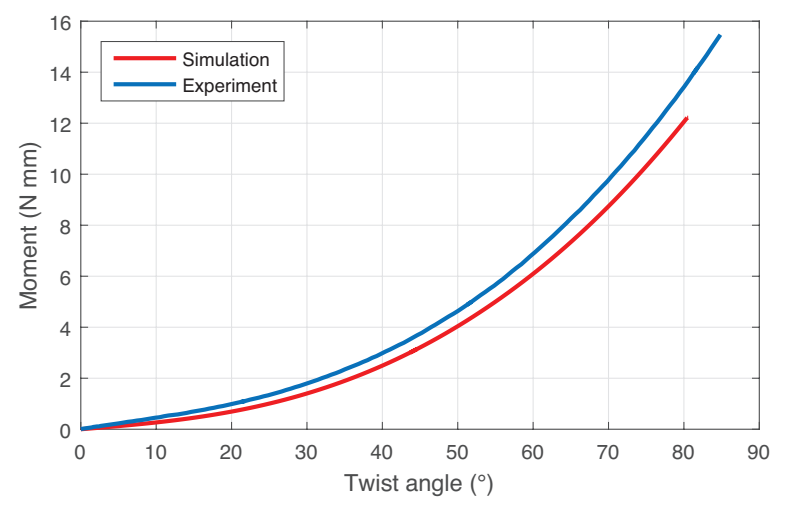

Figure 9. Comparison of torsion results from simulation to experiment. 
Consider bending around the $\mathrm{X}$-axis. The stiffness obtained experimentally closely matches the simulation result, Figure 8(a). The experimental behavior for negative moments closely matches the simulation. However, the behavior for positive moments differs past a certain point. Both curves start linearly, but while the numerical curve has a sharp corner at buckling, the experimental curve has instead a smooth transition from one regime to the other. From the experiments, the maximum moment that the TRAC boom can withstand before final buckling is $0.5 \mathrm{~N} \mathrm{~m}$ with a positive moment, and $0.4 \mathrm{Nm}$ with a negative moment.

Figure 8(b) shows the comparison for bending around the Y-axis. Because of small defects in the TRAC boom, small kinks appeared in the structure at the beginning of the experiment, as shown in Figure 10. For this reason, the boom never entered the initial linear regime, instead going immediately into a partially buckled stable regime until final buckling occurred. Therefore, the initial bending stiffness is lower than expected from the simulation, but closely matches the bending stiffness post-buckling in simulation, which corresponds to the stable buckled regime. For Y-axis bending, final buckling occurs when a moment of 0.26 $\mathrm{Nm}$ is applied.

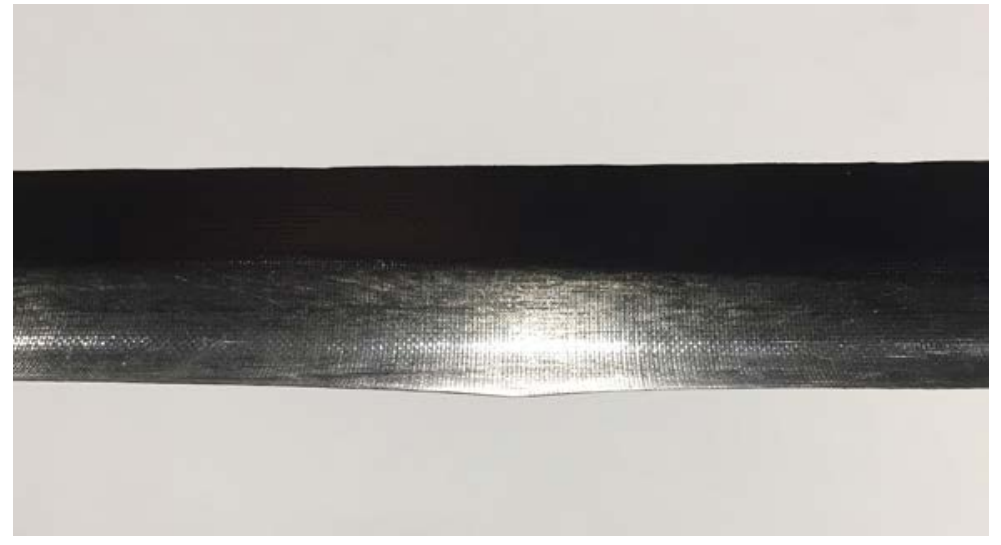

Figure 10. Kink appearing at a load of $0.027 \mathrm{Nm}$ (Sample 3).

The open, thin-walled section of the TRAC boom leads to low torsional stiffness. However, the tests have shown that very large deformation can be achieved without any buckling of the structure. Figure 9 shows the comparison between the numerical simulation and the experimental data. While the experimental behavior closely matches the simulation results, we can observed that the test results display a slightly higher torsional stiffness. Over the range of $5^{\circ}$, it translates into a factor of approximately 2 in torsional stiffness.

\section{Coiling Behavior}

TRAC booms can be wrapped tightly around a cylindrical hub. To wrap long booms, the case $R \gg r$ is of interest. In this case, a tension force is required for the TRAC booms to conform to the wrapped radius $R$, otherwise localized bends of radius $r$ form instead. ${ }^{5}$ These bends have the potential to cause problems during deployment, including jamming and higher required deployment forces. The coiling of a TRAC boom was simulated and also investigated experimentally, to capture both the qualitative behavior and the force profile required to tightly wrap such a boom.

The radius of the localized bend can be estimated from minimizing the energy in the two flanges and bond region, through an analysis similar to that of the localized bend radius in a tape spring. ${ }^{9}$ The energy in the transition region either side of the localized bend can be neglected, as it will be identical for any bend radius. This holds unless other localized bends or a clamped boundary condition are close enough to begin interacting with each other. The bending energy in both the flange and the bond region can be calculated from

$$
U_{\text {bending }}=\left(D_{11} \Delta \kappa_{t}^{2}+2 D_{12} \Delta \kappa_{t} \Delta \kappa l+D_{22} \Delta \kappa_{l}^{2}\right) R \alpha s
$$

where the $R \alpha s$ term is the total area of localized bend. $D_{11, i}, D_{12, i}$ and $D_{22, i}$ correspond to the elements in the D matrix that relate curvature to the bending moment in the composite shell. Note that $i=f 1, f 2$ or $B$ for the inner flange, outer flange and bond region respectively.

For the flanges $\Delta \kappa_{l}= \pm \frac{1}{R}, \Delta \kappa_{t}=\frac{1}{r_{\text {boom }}}$ and $s=\theta r_{\text {boom }}$. For the bond region, $\Delta \kappa_{l}=\frac{1}{R}, \Delta \kappa_{t}=0$ and $s=w$, the height of the bond region. Taken together, the total bending energy in the localized bend is given 
by

$$
U_{\text {total }}=\operatorname{R\alpha r}_{\text {boom }} \theta\left(\left(\frac{D_{11, f 1}}{R^{2}}+2 \frac{D_{12, f 1}}{R r_{\text {boom }}}+\frac{D_{22, f 1}}{r_{\text {boom }}^{2}}\right)+\left(\frac{D_{11, f 2}}{R^{2}}-2 \frac{D_{12, f 2}}{R r_{\text {boom }}}+\frac{D_{22, f 2}}{r_{\text {boom }}^{2}}\right)\right)+\frac{D_{11, b}}{R^{2}} R \alpha w
$$

Now, since the flange layups are equal and symmetric, $D_{11, f 1}=D_{11, f 2}, D_{12, f 1}=D_{12, f 2}, D_{22, f 1}=D_{22, f 2}$ and the equation simplifies to:

$$
U_{\text {total }}=2 R \alpha r_{\text {boom }} \theta\left(\frac{D_{11, f}}{R^{2}}+\frac{D_{22, f}}{r_{\text {boom }}^{2}}\right)+\frac{D_{11, b}}{R^{2}} R \alpha w
$$

Differentiating with respect to $\mathrm{R}$ and setting equal to zero gives the minimum energy condition, which leads to an estimate of the localized bend radius for a TRAC boom made entirely from symmetric layups:

$$
R=\sqrt{\frac{2 r_{\text {boom }} \theta D_{11, f}+w D_{11, b}}{2 D_{22, f}} \frac{r_{\text {boom }}}{\theta}}
$$

\section{IV.A. Numerical Analysis of Coiling Behavior}

To simulate coiling LS-Dyna, a finite element solver that uses explicit integration, was selected. This solver was selected because of its robust contact algorithm and ability to capture any dynamics that occur during coiling.

The finite element model is shown in Figure 11. It consists of a fully deployed TRAC boom, four temporary cylinders of diameter $d_{c y}=40 \mathrm{~mm}$ used to create the initial two folds, and a rigid, cylindrical hub of diameter $d_{h u b}=165 \mathrm{~mm}$. The temporary cylinders and hub were modeled as rigid bodies, fully constrained in all degrees of freedom. The TRAC boom was meshed with $C^{0}$ triangular shell elements. Contact is applied through LS-Dyna's automatic surface to surface and automatic single surface keywords. The friction coefficient obtained experimentally was set at $\mu=0.195$. The flange thickness was set as 0.071 $\mathrm{mm}$, and the bond region thickness double that at $0.142 \mathrm{~mm}$. The carbon-fibre layup and ply properties were the same as detailed in Section II. The flange cross section has radius $r_{\text {boom }}=10.8 \mathrm{~mm}$ and subtended angle $\theta=104^{\circ}$. These correspond to average values of the boom tested later in Section IV.B. With these parameters, $D_{11, f}=0.0033 \mathrm{~N} \mathrm{~m}, D_{22, f}=0.00065 \mathrm{~N} \mathrm{~m}$ and $D_{11, b}=0.019 \mathrm{~N} \mathrm{~m}$. For these values, Equation 6 predicts a localized bend radius $R=36 \mathrm{~mm}$.

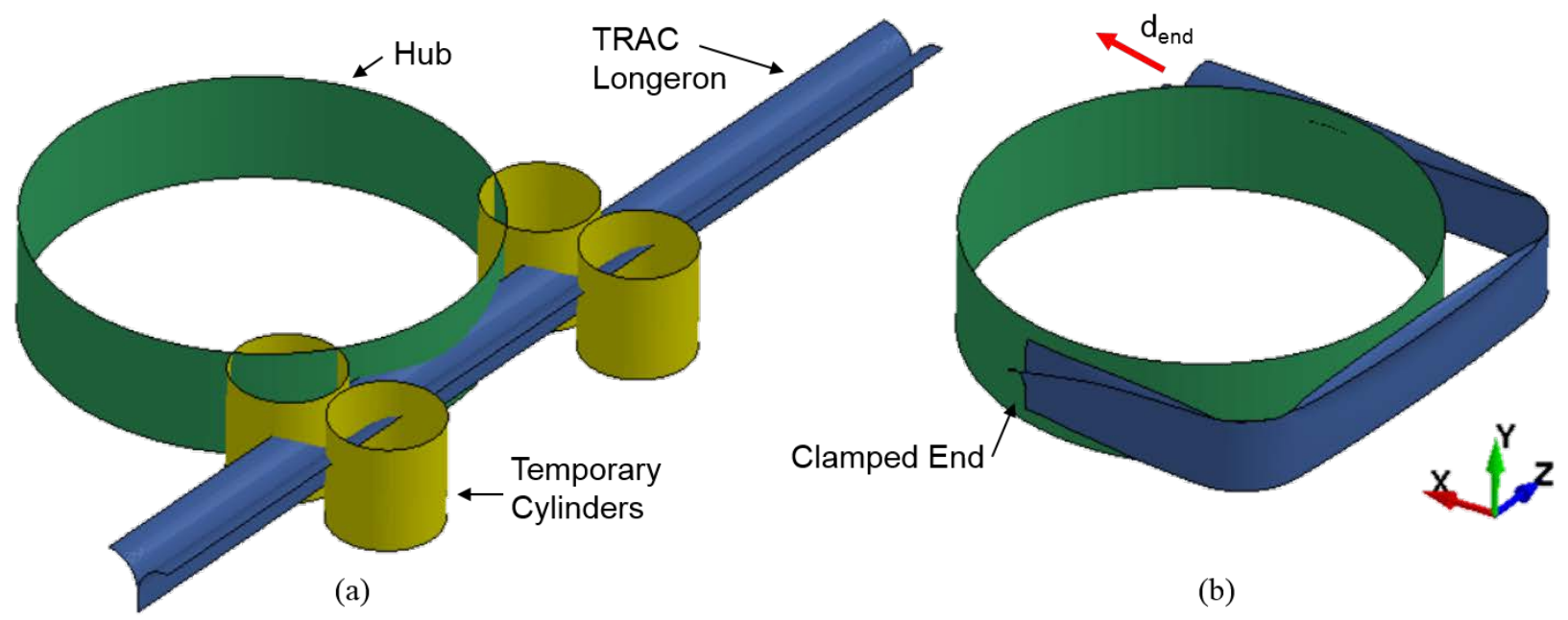

Figure 11. (a) Initial configuration of coiling numerical simulation. (b) boom folded with two localized bends and temporary cylinders removed. Displacement $d_{e n d}$ applied to fully coil Boom.

\section{IV.A.1. Folding Step}

The simulation started with the boom in the fully deployed configuration, shown in Figure 11(a). To fold the boom into the initial two-fold configuration, shown in Figure 11(b), boundary conditions were applied to 
the flange edges to flatten the flanges at the two fold locations. After flattening, these boundary conditions were removed, and contact with the four cylinders was enabled. These cylinders force the TRAC boom to remain flattened. Displacement boundary conditions were then applied to the ends of the boom, completely constraining the motion of the ends as they were wrapped around two temporary cylinders.

Contact with the temporary cylinders was then removed, and the TRAC boom was allowed to find an equilibrium configuration. Damping was applied to remove excess kinetic energy. This results in the two-fold configuration in Figure 11(b).

\section{IV.A.2. Coiling Results}

As shown in Figure 11(b), one end of the boom was clamped, and displacement boundary conditions applied to the other end. A plot of the resulting tension force as a function of the end displacement, $d_{\text {end }}$, is shown in Figure 12. Key TRAC boom configurations obtained during coiling are shown in Figure 13.

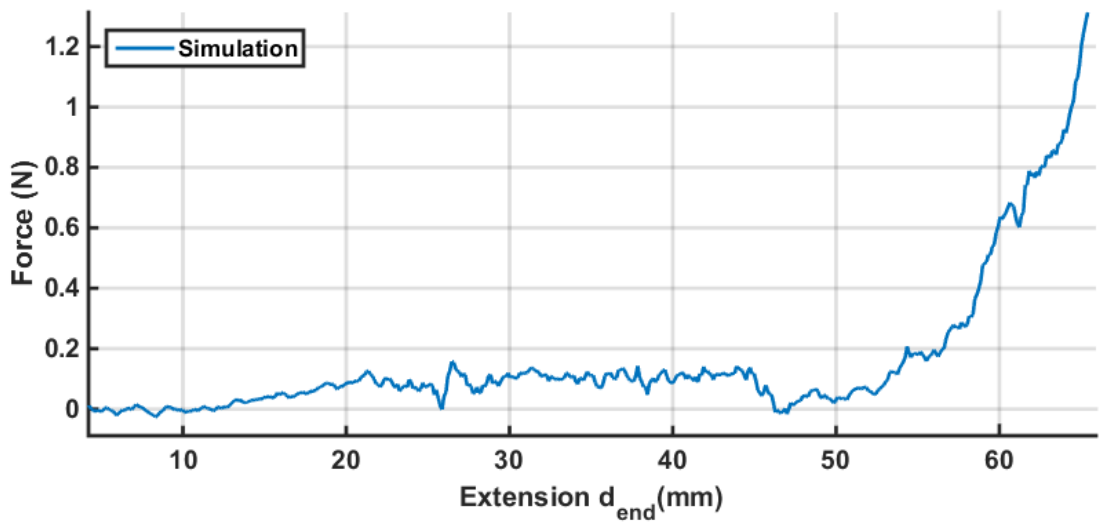

Figure 12. Simulation results for a carbon-fibre TRAC longeron with constant cross-section and bond region thickness of $0.142 \mathrm{~mm}$

As expected, the initial TRAC boom equilibrium state has two localized hinges as seen in Figure 13(a). As displacement is applied to one side, the localized bends increase in radius, see Figure 13(b). As the bend radii get bigger, the inner flange opens while the outer flange continues to conform to the localized bend radius. At $d_{\text {end }}=41.7 \mathrm{~mm}$ the bottom fold bifurcates into two. This trend continues, with another bifurcation at $d_{\text {end }}=48.5 \mathrm{~mm}$. These bifurcations are reflected in the force plot shown in Figure 12. The sharp jumps correspond to fold bifurcations. The coordinates of the bond region nodes were extracted and used to calculate the localized bend radius at $d_{\text {end }}=0$, corresponding to Figure 13(a). In the simulation $R$ ranged from 35.4 to $39 \mathrm{~mm}$ which compares favorably with the analytical prediction of $36 \mathrm{~mm}$. The radius of curvature was lowest near the edges of the localized bend, and highest in the middle.

\section{IV.B. Coiling Experiments}

To validate the simulations in Section IV.A, a cylindrical hub of diameter $d_{h u b}=165 \mathrm{~mm}$ was rigidly attached to the base of an Instron materials testing machine. One end of a composite TRAC boom was clamped to the side of the hub, and folded in around the hub, as shown in Figure 14. The other end was attached to the load cell of the testing machine. The force was recorded as this end was displaced vertically at a rate of $30 \mathrm{~mm} / \mathrm{min}$.

The experimental results highlight two aspects. First, the initial folded configuration is non-unique, and depends on exactly how the fixed end is clamped, and upon the method of folding. In Figure 14(a) there are four localized fold initially. In Figure 14(c) there are three and in Figure 14(e) there are only two. In Figure 14(a) and 14(b) the first localized bend is hidden from the camera and is near the clamped end. The effect of the number of localized folds is evident in the corresponding force plots.

For all initial configurations, the tension force required is almost zero until the inner flange in-between the localized folds comes into contact with the hub. At this point the tension force rapidly increases, which corresponds to $d_{\text {end }}=48.2 \mathrm{~mm}, 41.4 \mathrm{~mm}$ and $38.8 \mathrm{~mm}$ for the four-fold, three-fold and two-fold initial configurations respectively. In the two- and three-fold initial configurations, the distance between localized 


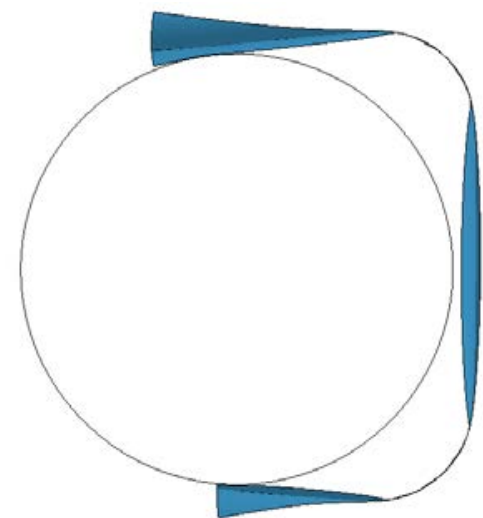

(a) $d_{\text {end }}=0$ Initial two-fold configuration

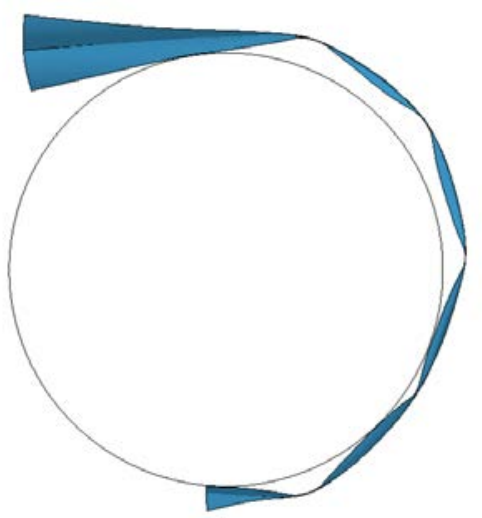

(d) $d_{\text {end }}=48.5 \mathrm{~mm}$, configuration after bifurcation to five folds

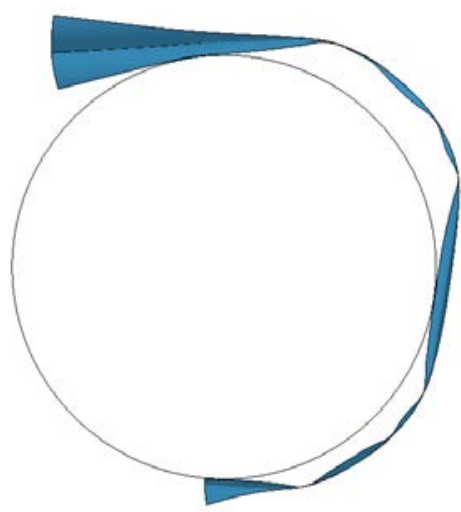

(b) $d_{\text {end }}=38.6 \mathrm{~mm}$, configuration just before bottom fold bifurcates

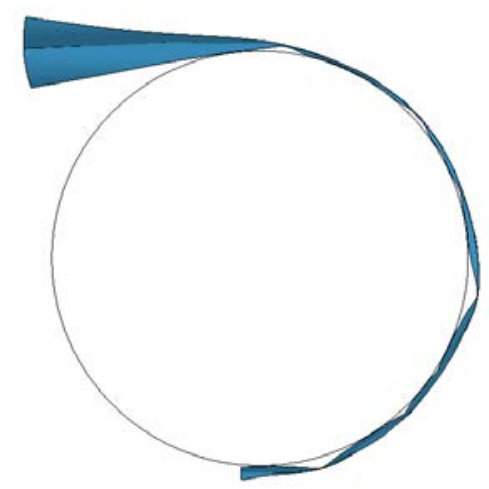

(e) $d_{\text {end }}=64.7 \mathrm{~mm}$, flanges start to flatten instead of the localized folds bifurcating

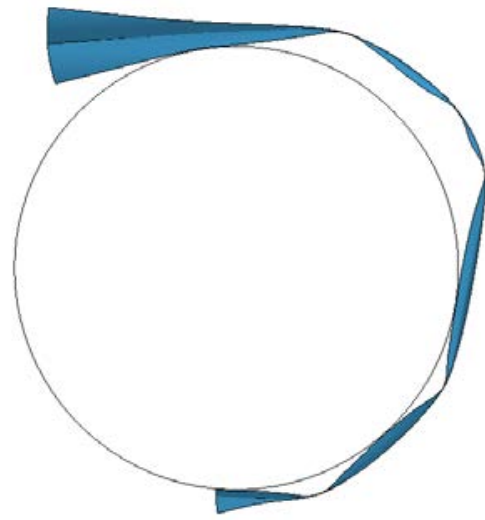

(c) $d_{\text {end }}=41.7 \mathrm{~mm}$, bottom fold has bifurcated into two.

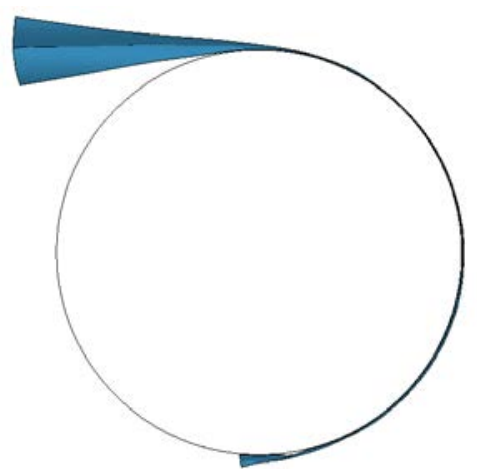

(f) $d_{\text {end }}=71.8 \mathrm{~mm}$, fully coiled configuration

Figure 13. Localized fold configurations that develop during the coiling of boom with constant cross-section and bond region thickness of $0.142 \mathrm{~mm}$. 


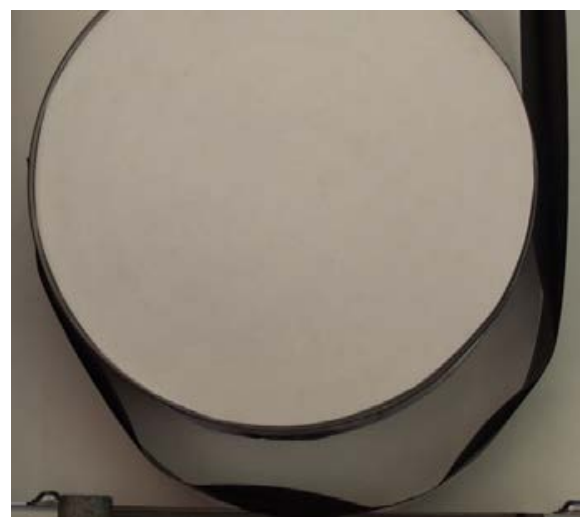

(a) Four fold initial configuration

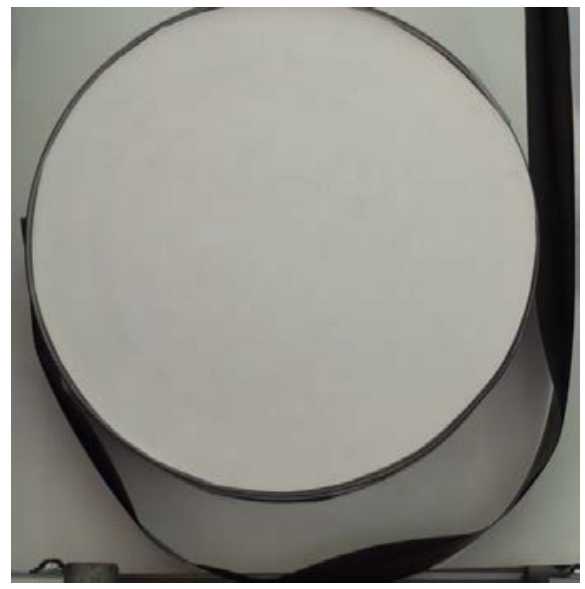

(c) Three fold initial configuration

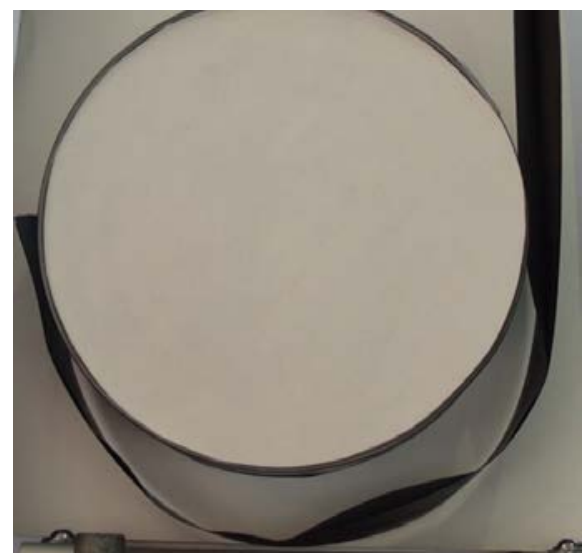

(e) Two fold initial configuration

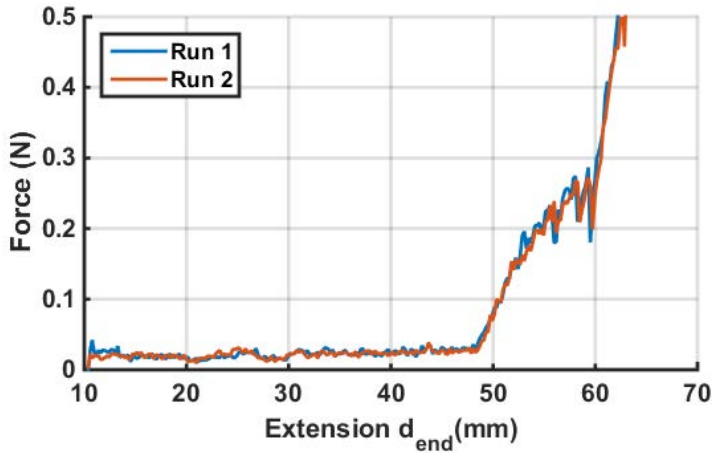

(b) Experiment forces corresponding to four fold initial configuration

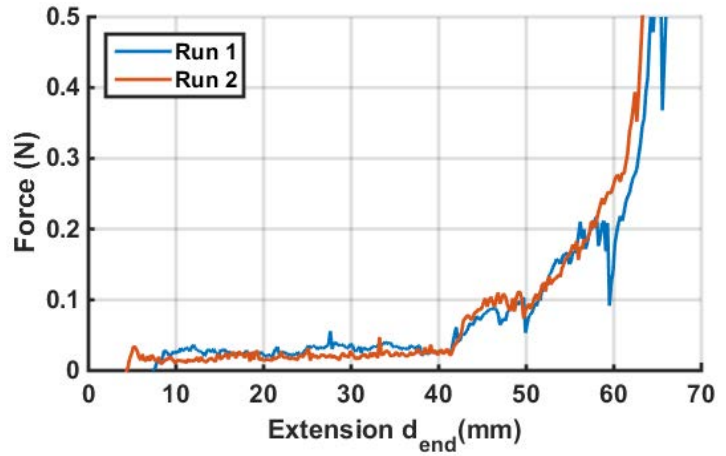

(d) Experiment forces corresponding to three fold initial configuration

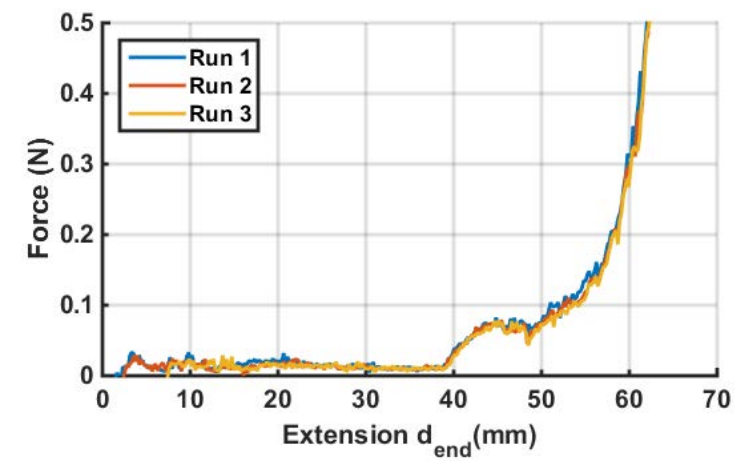

(f) Experiment forces corresponding to two fold initial configuration

Figure 14. Left: Initial TRAC boom experimental folded configurations. The left end of the TRAC boom is rigidly mounted to the cylindrical hub, and the right end to an Instron tensile testing machine. Subtle changes in the clamped boundary condition or folding process lead to different initial configurations. Right: Corresponding force profiles obtained during coiling. 
folds is greatest, allowing the inner flange to open more and therefore come into contact with the hub sooner than the four-fold initial configuration. In two fold initial configuration, the inner flange is compressed against the hub in only one place leading to a force plateau at $0.08 \mathrm{~N}$. In the three fold initial configuration the inner flange is in contact with the hub in two locations, and the resulting plateau is at $0.1 \mathrm{~N}$.

The two-fold initial configuration is the closest to that modeled in LS-Dyna. Both the simulation and the experiment exhibit a force plateau, although in the experiment the plateau was measured at $0.08 \mathrm{~N}$, compared with $0.1 \mathrm{~N}$ in the simulation. In addition however, the simulation force increases from zero at $d_{\text {end }}=12.7 \mathrm{~mm}$, much earlier than in the experiment at $d_{\text {end }}=38.8 \mathrm{~mm}$. Finally, at $d_{\text {end }}=0 \mathrm{~mm}$ the localized folds in the simulation are much smaller than in the experiments. From Equation 6 we know this means that either the bond region width and/or the thickness are higher.

\section{IV.C. Implementing Imperfect TRAC Boom Geometry}

One reason the initial simulation did not accurately capture the experimental behavior is that the model did not account for any imperfections. The laser scanner on a FaroArm portable coordinate measuring machine was used to obtain a point cloud of the TRAC boom surface. The point cloud data also captured initial boom displacement in the Y-direction, as shown in Figure 15(a). Additional measurements were taken with a micrometer to determine the thickness variation along the boom length. Figure 15(b) shows the thickness of the bond region as measured by a micrometer $2 \mathrm{~mm}$ and $6 \mathrm{~mm}$ from the edge of the bond region. With this information a new mesh was generated that included the initial Y displacement. In addition the simulation bond-region thickness was increased to $195 \mu \mathrm{m}$. The thicker bond region results in $D_{11, b}=0.044 \mathrm{~N} \mathrm{~m}$ and a localized bend of $R=47 \mathrm{~mm}$. The flange radii and subtended angle were left unchanged and constant along the boom length.

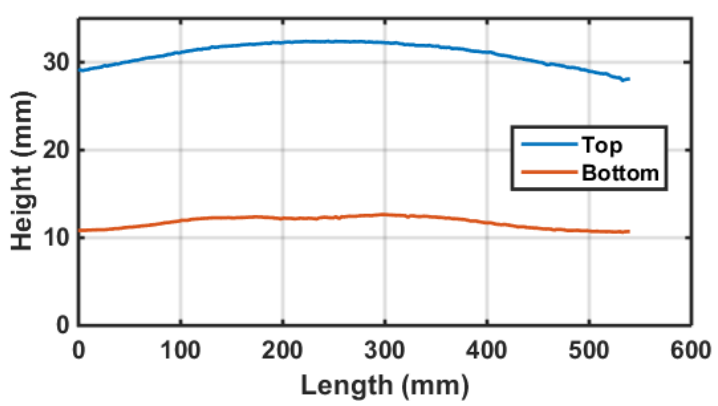

(a) Height of bond region when boom is resting on its flanges

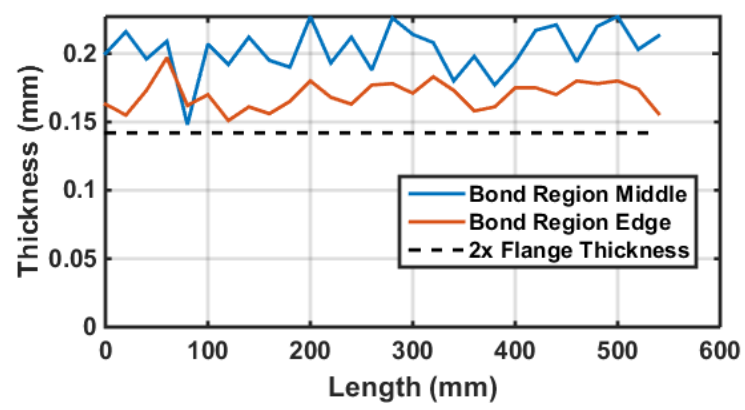

(b) Thickness of bond region along boom length

Figure 15.

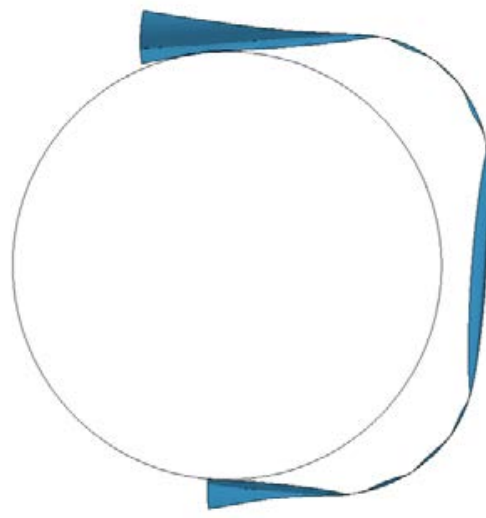

(a) Initial folded configuration

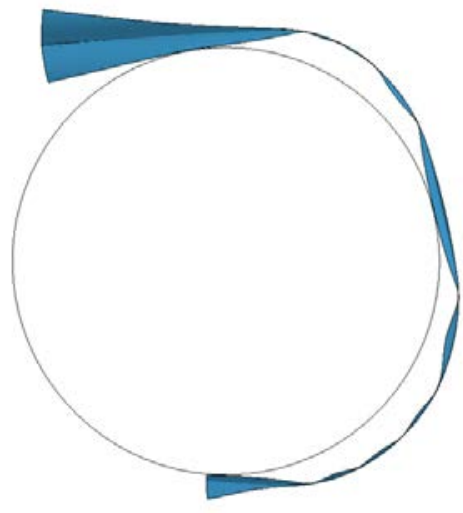

(b) $d_{\text {end }}=38.8 \mathrm{~mm}$, the two localized folds have increased in radii

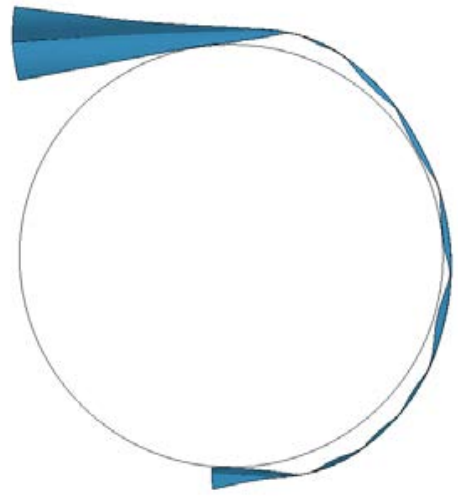

(c) $d_{\text {end }}=52.4 \mathrm{~mm}$,

Figure 16. Localized fold configurations that develop during coiling of boom with constant cross-section and bond region thickness of $0.195 \mathrm{~mm}$. 


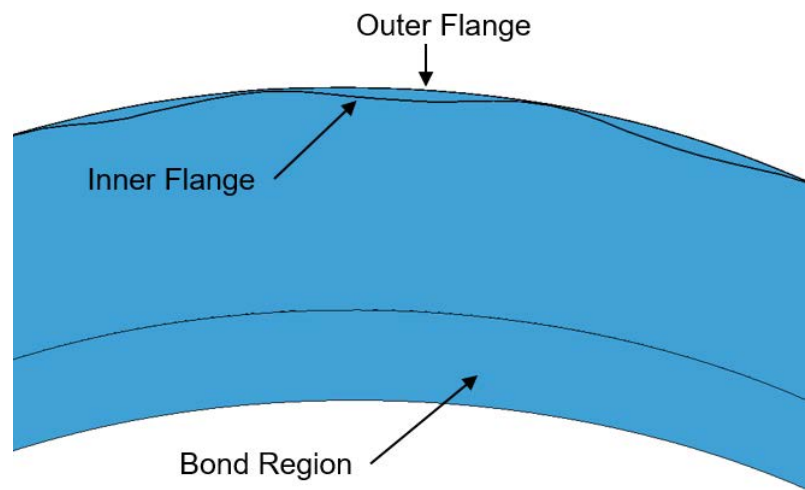

(a) Isometric view of the bottom localized fold in Figure 16(a)

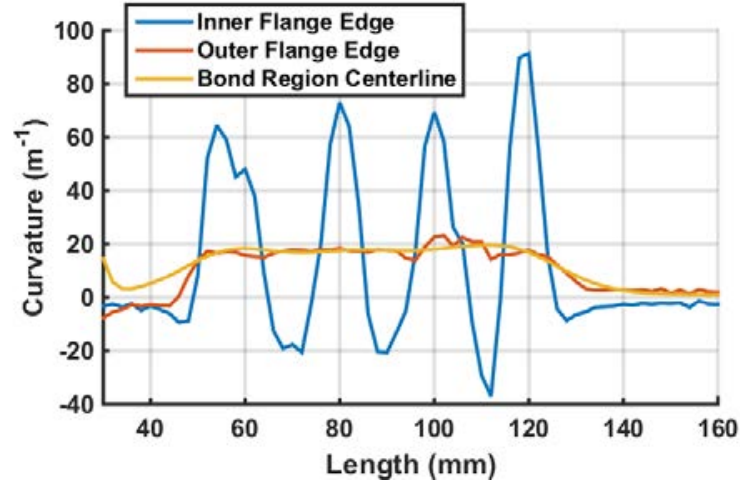

(b) Curvature of inner flange edge, outer flange edge and bond region centerline in the bottom localized fold in Figure 16(a). Fold ends at length $=130 \mathrm{~mm}$.

Figure 17.

Figure 18 is a plot of the force results from the modified simulations, together with the experimental data for the two-fold initial configuration. The inner flange between the two localized folds comes into contact at $d_{\text {end }}=28 \mathrm{~mm}$, an improvement of $15 \mathrm{~mm}$ compared to the previous simulation. Once the inner flange in the corresponding experiment comes into contact with the hub at $d_{\text {end }}=38 \mathrm{~mm}$ there is close agreement between the experiment and simulation. The simulation saw-tooth force profile before $d_{e n d}=28$ $\mathrm{mm}$ is a direct result of friction causing stick-slip behavior. The reason for the force plateau can be seen in Figure 16(b). The TRAC boom still has two localized folds which just increase in radius. The jumps in force between $d_{\text {end }}=28 \mathrm{~mm}$ and $d_{\text {end }}=52.4 \mathrm{~mm}$ correspond to both friction, and to the formation and bifurcation of smaller localized bends in just the inner flange. Finally, at $d_{\text {end }}=0$ the localized bend radius is $R=49$ to $56 \mathrm{~mm}$. This is larger than the estimate of $47 \mathrm{~mm}$. This is because even in the initial two fold configuration the inner flange is opening along the localized bend as seen in Figure 17(a), while Figure 17(b) clearly shows that the outer flange edge and bond region have almost identical curvature. The opening of the inner flange relieves some of the bending energy in this flange, and results in a slightly larger radius of curvature in the bond region.

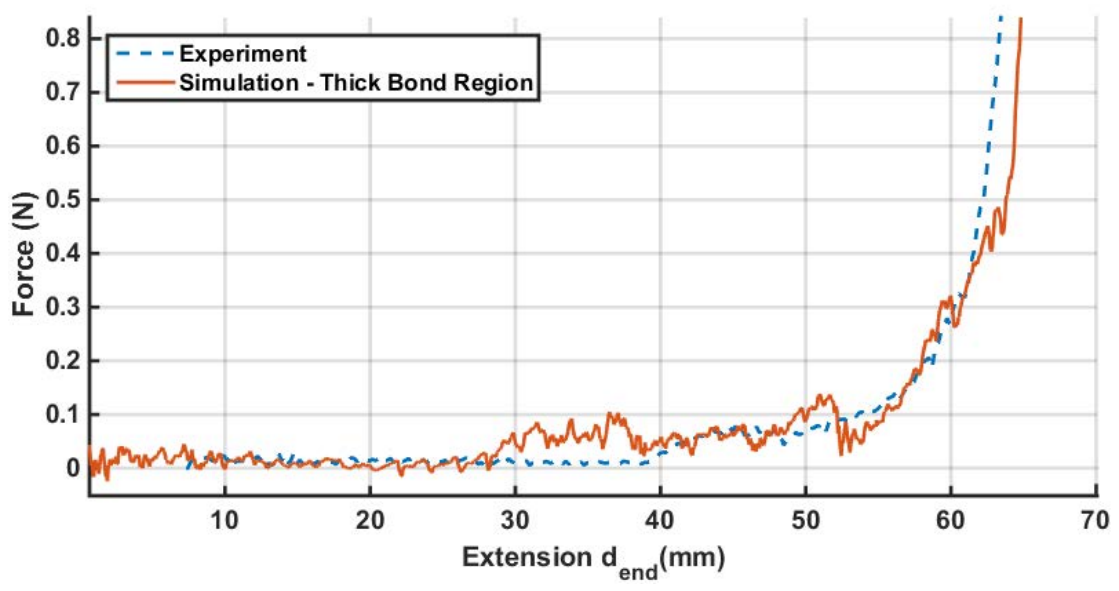

Figure 18. Comparison of experimental results with simulations incorporating manufacturing imperfections 


\section{Conclusion}

A characterization of ultra-thin TRAC booms has been presented. A new manufacturing process for ultra-thin $(71 \mathrm{\mu m})$ boom using epoxy reinforced carbon fiber prepregs was used to construct $0.55 \mathrm{~m}$ long prototypes. Both numerical simulations and experiments were used to characterize the deployed mechanical properties of the booms. LS-Dyna finite element simulations were used to accurately capture the non-linear behavior of booms wrapped around large radius hubs. In particular, as a TRAC boom is wrapped around a hub of radius significantly greater than its natural bend radius it forms a series of localized bends. The radii of these bends can be predicted analytically, although the prediction is less accurate when the natural radius of curvature of the TRAC boom is significantly larger than the transverse radii of the flanges. As the boom is coiled further the radii of the localized bends increase until they bifurcate. This continues until there are a large number of localized bends, whereupon the bifurcations cease and the inner flange slowly closes up until the boom is fully wrapped. Finally, the thickness of the bond region has a significant effect on both the force profile and the folded configuration of partially wrapped TRAC booms.

\section{Acknowledgments}

The authors acknowledge financial support from the Northrop Grumman Corporation, the Keck Institute for Space Studies and the Natural Sciences and Engineering Research Council of Canada.

\section{References}

\footnotetext{
${ }^{1}$ Murphey, T. W. and Banik, J., "Triangular rollable and collapsible boom," March 1 2011, US Patent 7,895,795.

${ }^{2}$ Banik, J. and Murphey, T. W., "Performance Validation of the Triangular Rollable and Collapsible Mast," Proceedings of the 24th Annual AIAA/USU Conference on Small Satellites, Logan, UT, 2010.

${ }^{3}$ Biddy, C. and Svitek, T., "LightSail-1 solar sail design and qualification," Proceedings of the 41st Aerospace Mechanisms Symposium, 2012.

${ }^{4}$ Whorton, M., Heaton, A., Pinson, R., Laue, G., and Adams, C., "Nanosail-D: the first flight demonstration of solar sails for nanosatellites," 22nd AIAA/USU Conference on Small Satellites, 2008.

${ }^{5}$ Seffen, K. and Pellegrino, S., "Deployment dynamics of tape springs," Proceedings of the Royal Society of London A: Mathematical, Physical and Engineering Sciences, Vol. 455, The Royal Society, 1999, pp. 1003-1048.

${ }^{6}$ Estvanko, B., Maji, A., and Ng, P., "Numerical analysis of a tape spring hinge folded about two axes," Earth and Space 2012@ sEngineering, Science, Construction, and Operations in Challenging Environments, ASCE, 2012, pp. 714-721.

${ }^{7}$ Walker, S. J. and Aglietti, G. S., "An investigation of tape spring fold curvature," Proceedings of the 6th International Conference on Dynamics and Control of Systems and Structures in Space, Citeseer, 2004.

${ }^{8}$ Stabile, A. and Laurenzi, S., "Coiling dynamic analysis of thin-walled composite deployable boom," Composite Structures, Vol. 113, 2014, pp. 429-436.

${ }^{9}$ Calladine, C., "The theory of thin shell structures 1888-1988," Proceedings of the Institution of Mechanical Engineers, Part A: Journal of Power and Energy, Vol. 202, No. 3, 1988, pp. 141-149.
} 